\title{
FUZZY LOGIC APPROACH TO ESTIMATING TENDENCIES IN TARGET BEHAVIOR
}

\author{
Albena TCHAMOVA and Tzvetan SEMERDJIEV
}

\section{Introduction}

Angle-only tracking systems based on passive sensors are poorly developed due to a number of complications. They receive signals transmitted from other emitters and tend to be less precise than those based on active sensors. However, one important advantage is their vitality of being stealth. In general, passive sensors make only lineof-sight angle detection. In the single sensor case that means that we know only direction of the target as an axis, but the true target position and behavior (approaching or descending) remain unknown. The problem of determining an objects' position without using measurements of the distance to it concerns moving platform applications, astronomy and some military situations, where it is important to estimate the position (respectively the distance to the object) and, in particular, the behavior of moving targets. In military avionics, for example, some fighter defending against a raid may wish to launch a missile as a counteraction to the enemy, but it could not do this until the position and the behavior of the opposing target are not known. In such situations, the uncertainty with respect to the opposite target behavior requires to compensate the missing range by utilizing the extracted from the received emitter's signal attributes. This information can be used to assess tendencies in target's behavior and its location and, consequently, to improve the overall angle-only tracking performance.

The objective of this work is to present an approach for target behavior tendency estimation, based on the application of the principles of fuzzy logic to conventional passive radars. It utilizes the measured emitter's amplitude values in consecutive time moments and uses a set of particular filters design with respective set of possible target behavior models. In real world situations, fuzzy logic provides an approximate but consistent solutions to complex engineering problems, where numerical data usually are noisy and incomplete, and the linguistic information is imprecise and vague. Compared to

INFORMATION \& SECURITY. An International Journal, Vol. 9, 2002, 58-69 
other methods such as Bayesian and Evidential Reasoning, Fuzzy Logic shows some important advantages: it is suitable and well adapted to use uncertain data and it is a much more expressive tool for codification of expert knowledge; measurement errors are explicitly taken into account; it entails modest computational load and provides decisions in a simple and robust way.

\section{Statement of the Problem}

In order to track targets using passive sensors it is necessary to compensate the unknown ranges by using additional information received from the emitter. In our case, we assume that the observed target emits constant signal. It is received by the sensor with a non-constant, but a varying strength (referred to as amplitude). The augmented measurement vector at the end of each time interval $k=1,2, \ldots$ is $Z=\left\{Z_{\Theta}, Z_{A}\right\}$, where: $Z_{\Theta}$ denotes the measured local angle with zero-mean Gaussian noise $\nu_{\Theta}$, and $Z_{A}=A+\nu_{A}$ denotes corresponding amplitude value with zero-mean Gaussian noise $\nu_{A}=N\left(0, \sigma_{\nu_{A}}\right)$ and covariance $\sigma_{\nu_{A}}$. The variation of the amplitude value is caused by the cluttered environment and the varying unknown distance to the object. It is conditioned by possible modes of target behavior (approaching or descending). Our goal is to utilize received amplitude feature measurements for predicting and estimating the tendency of target behavior.

The block diagram of target behavior tracking system is shown on Figure 1. Two single-model-based filters running in parallel and using two models for target behavior (Approaching and Receding) are maintained. At the initial moment $k$ the target is characterized by the fuzzified amplitude state estimates according to the two models $A^{A p p}(k / k)$ and $A^{\operatorname{Rec}}(k / k)$. The new observation at time $k+1$ is assumed to be the true value, corrupted by additive measurement noise. It is fuzzified according to the chosen fuzzification interface.

In order to reduce the influence of measurement noise, a weighting procedure is developed and applied. Particular tendency prediction and updating methods are used to estimate present and future target behavior. In general, this diagram resembles the commonly used approaches in standard tracking systems. ${ }^{1,2}$ The peculiarity is the implemented fuzzy logic approach ${ }^{3,4,5}$ in the realization of the main steps of the procedure.

\section{Basic Elements of Fuzzy Logic Systems}

In order to resolve the stated problem we apply fuzzy logic as a framework for simultaneous processing and handling of numerical and linguistic data to obtain consistent representation of target behavior in a timely manner. Fuzzy systems differ from classical mathematical-model ones. They do not require strong mathematical models 


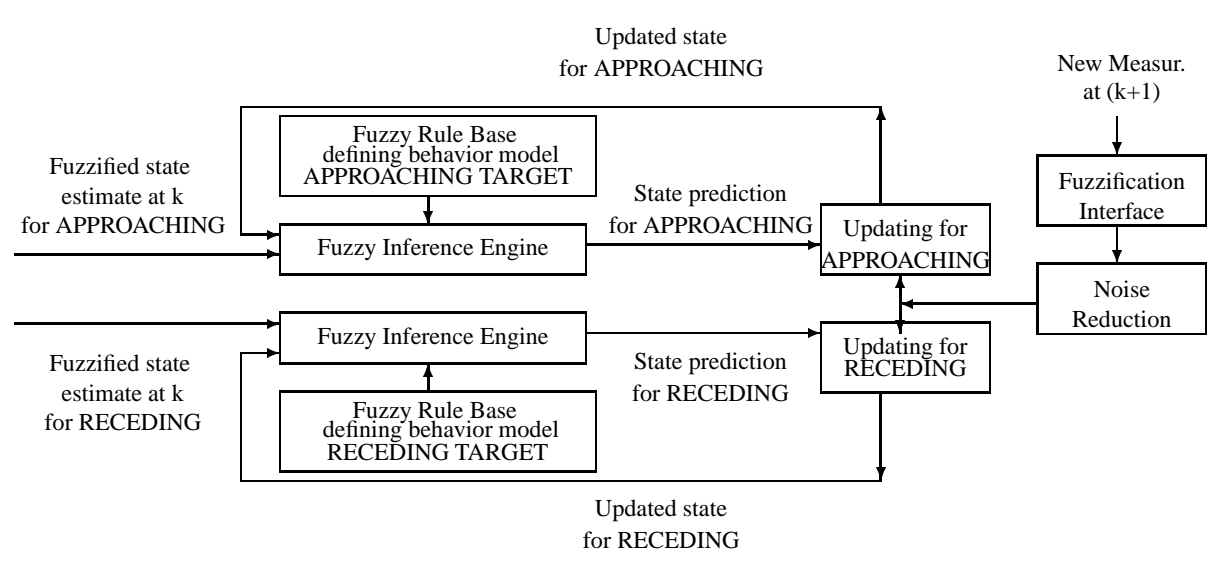

Figure 1: Block diagram of target's behavior tracking system

of functional dependency between system's input and output. Mathematical models of system states and measurement processes restrict the range of real-world applications, because of difficulties in the incorporation of nonmathematical knowledge. Basically, fuzzy logic systems ${ }^{3}$ consist of a set of fuzzy associative memory rules or (input,output) associations, operating in parallel, to various degrees. Fuzzy logic systems transform crisp or fuzzy set inputs into a crisp or fuzzy-set output. Further in this section we describe the basic elements in fuzzy reasoning: fuzzy sets, fuzzification interface, fuzzy knowledge base, inference engine and identification of fuzzy models.

\subsection{Fuzzy Sets}

Fuzziness is a condition, which relates to classes whose boundaries are unsharply defined. A fuzzy set $F$ is a generalization of an ordinary set by allowing a degree of membership for each element. It is defined on a universe of discourse $U$. The membership function $\mu_{F}(x)$ provides a measure of degree of similarity of an element in $U$ to the fuzzy subset and takes its values in the interval $[0,1]$. Each fuzzy set represents a linguistic value of some linguistic variable. It is defined as a variable whose values are sentences in a natural language. The determination of fuzzy membership functions is the most important issue in applying fuzzy system approach to engineering problems. No common approach is available for determining these functions. In some cases, they are attained subjectively as a model for human concepts. In other cases, they are based on statistical or/and empirical distributions, on heuristic determination, on reliability with respect to some particular problem, or on theoretical demands. In any case, the definition of membership functions is not arbitrary. 


\subsection{Fuzzification Interface}

Fuzzification refers to replacing a crisp set with a set whose boundaries are fuzzy. It transforms each numerical measurement received from a sensor into fuzzy set according to the a priori defined fuzzy partition of input space - the frame $\theta$. This frame comprises all considered linguistic values related to particular important input variables and their membership functions. It is well known,${ }^{4}$ that much of the evidence on which human decisions are based is fuzzy. Because of that fact, the fuzzification of numerical sensory data needs dividing an optimal membership into a suitable number of fuzzy sets. Such division provides smooth transitions and overlaps among the associated fuzzy sets according to the particular real world situation.

\subsection{Fuzzy Knowledge Base}

Fuzzy IF-THEN rules provide a methodology to represent some objective and/or human knowledge. From this point of view, each fuzzy rule is a scheme for capturing knowledge that involves imprecision. The principle feature of fuzzy rule-based reasoning is its partial matching capability. It makes possible an inference to be made from a fuzzy rule even when the rule's condition is partially satisfied. Fuzzy mapping rules describe a functional mapping relationship between inputs (antecedents) and output (consequent) using linguistic terms.

The foundation of fuzzy mapping rules is a fuzzy graph $g$, which is an union of Cartesian products involving linguistic input-output associations. It is described by a set of $i$ number fuzzy rules in the form of: 'IF $x$ is $A_{i}$ THEN $y$ is $B_{i}$ '. This is expressed mathematically as:

$$
g=\bigcup_{i} A_{i} \times B_{i}
$$

where $A$ and $B$ are the linguistic values, describing input and output variables. The Cartesian product of $A$ and $B$ is defined as:

$$
\mu_{A \times B}(u, v)=\mu_{A}(u) \otimes \mu_{B}(v),
$$

where $\otimes$ denotes a fuzzy conjunction (t-norm) operator; $\mu_{A \times B}(u, v)$ is a membership function, which measures the degree of truth of the implication relation between corresponding antecedents and consequents.

\subsection{Fuzzy Inference Engine}

Fuzzy mapping rules are designed as a group. The inference of such a collection is based on compositional rule of inference:

$$
B^{\prime}=A^{\prime} \circ g=A^{\prime} \circ \bigcup_{i} A_{i} \times B_{i} \text {. }
$$


Here $g$ represents the fuzzy graph of a given fuzzy model and the operator $\circ$ denotes the rule. It is not uniquely defined. By choosing different fuzzy conjunction and disjunction operators, one can get different representations.

\subsection{Fuzzy Model Identification}

A set of fuzzy mapping rules forms a fuzzy model. Depending on the choice of aggregation operator at the outputs of the fuzzy rules, fuzzy models can be classified into two categories: nonadditive and additive ones. The first group aggregates the outputs of fuzzy rules using the maximum operator, while the second uses an additive operator. Another important point is the appropriate mathematical interpretation of the t-norm operator in equation (2). There are multiple choices available, but it is proven,${ }^{3}$ that minimum and product inferences are most widely used in engineering applications, since they preserve the cause and effect relationship - the cornerstone principle of each modeling process. Relying on that, the inference scheme of the implemented particular fuzzy model is derived as a fuzzy graph, in which Larsen product operator is used for fuzzy conjunction and "maximum" for fuzzy union operator:

$$
g=\max _{i}\left(\mu_{A_{i} \times B_{i}}(u, v)\right)=\max _{i}\left(\mu_{A_{i}}(u) \cdot \mu_{B_{i}}(v)\right) .
$$

The inference is based on the most commonly used Zadeh max-min compositional rule. ${ }^{3,4}$ If input " $x$ is $A^{\prime \prime}$ " is given, the inferred output is:

$$
\mu_{B^{\prime}}(y)=\max _{x_{i}}\left(\min \left(\mu_{A^{\prime}}\left(x_{i}\right), \mu_{A \times B}\left(x_{i}, y_{i}\right)\right)\right)
$$

\section{Fuzzy Approach to Tracking Target Behavior}

There are a few basic components in the block diagram of the system for target behavior tracking, shown on Figure 1. In general, this diagram resembles the approaches commonly used in standard tracking systems. This section provides additional information on the specific implementation of the fuzzy logic approach to realize the main steps of tracking.

\subsection{Fuzzification Interface Determination}

An important variable in the particular case is the amplitude. Its values $A(k)$ are transmitted from the emitter and received at consecutive time moments $k=1,2, \ldots$. The fuzzification interface presented on Figure 2 maps $A(k)$ into four fuzzy sets:

$\Theta=\{\operatorname{Very} \operatorname{Small}(V S), \operatorname{Small}(S), \operatorname{Big}(B), \operatorname{Very} B i g(V B)\}$, which define the corresponding linguistic values related to the linguistic variable 'Amplitude Strength.' Their 


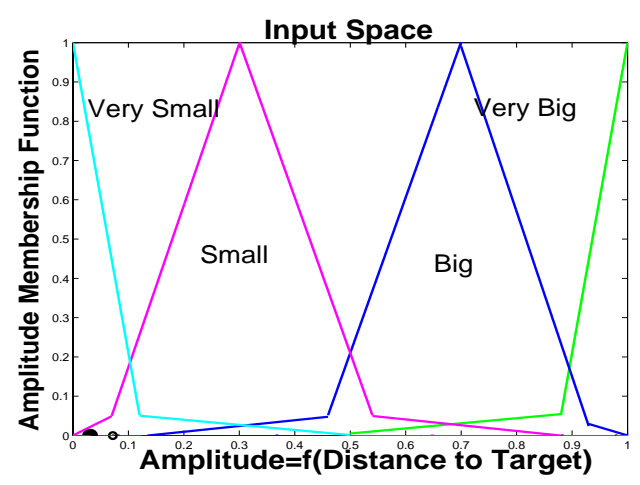

Figure 2: Fuzzification interface

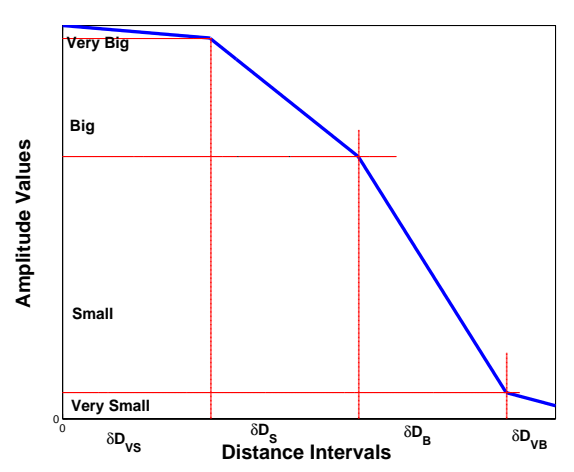

Figure 3: $A=f\left(1 / \delta_{D}\right)$

membership functions are not arbitrarily chosen, but rely on the well-known inverse proportion dependency between the measured amplitude value and the corresponding distance to the observed target (Figure 3).

The length of fuzzy sets' bases provides a design parameter which is calibrated to achieve satisfactory performance. Membership functions are tuned in conformity with the particular dependency $A=f\left(1 / \delta_{D}\right)$ which is a priori information. The degree of overlap between adjacent fuzzy sets reflects amplitude gradients in the boundary points of specified distance intervals $\delta_{D}$.

\subsection{Identification of Implemented Fuzzy Models}

In conformity with the core of our task, fuzzy rules' definition is consistent with the tracking of amplitude changes in consecutive time moments $k=1,2, \ldots$. A particular feature in this regard is that the considered fuzzy rules have one and the same antecedents and consequents. We define their meaning by using the linguistic terms and associated membership functions prespecified in paragraph 4.1. We consider two essential models of possible target behavior:

- Approaching Target. Its behavior in time is characterized as a stable process of gradual increase of the amplitude value that can be described by a set of transitions: $V S \rightarrow V S \rightarrow S \rightarrow S \rightarrow B \rightarrow B \rightarrow V B \rightarrow V B$;

- Receding Target. Its behavior in time is characterized as a stable process of gradual decrease of the amplitude value, that is described by a set of transitions: $V B \rightarrow$ $V B \rightarrow B \rightarrow B \rightarrow S \rightarrow S \rightarrow V S \rightarrow V S$.

To comprise appropriately these models, the following fuzzy rule bases have to be carried out: 
Behavior 1: APPROACHING TARGET

Rule1:IF $A(k)$ is $V S$ THEN $A(k+1)$ is $V S$ Rule2:IF $A(k)$ is $V S$ THEN $A(k+1)$ is $S$

Rule3:IF $A(k)$ is $S$ THEN $A(k+1)$ is $S$

Rule4:IF $A(k)$ is $S$ THEN $A(k+1)$ is $B$

Rule5:IF $A(k)$ is $B$ THEN $A(k+1)$ is $B$

Rule6:IF $A(k)$ is $B$ THEN $A(k+1)$ is $V B$

Rule7:IF $A(k)$ is $V B$ THEN $A(k+1)$ is $V B$
Behavior 2: RECEDING TARGET

Rule1:IF $A(k)$ is $V B$ THEN $A(k+1)$ is $V B$ Rule2:IF $A(k)$ is $V B$ THEN $A(k+1)$ is $B$

Rule3:IF $A(k)$ is $B$ THEN $A(k+1)$ is $B$

Rule4:IF $A(k)$ is $B$ THEN $A(k+1)$ is $S$

Rule5:IF $A(k)$ is $S$ THEN $A(k+1)$ is $S$

Rule6:IF $A(k)$ is $S$ THEN $A(k+1)$ is $V S$

Rule7:IF $A(k)$ is $V S$ THEN $A(k+1)$ is $V S$

In conformity with theoretical considerations and mathematical interpretations in paragraphs 3.4 and 3.5 and by using the specified membership functions, we obtain the resulting fuzzy graphs as fuzzy relations:

Relation1:Approaching Target

Relation2:Receding Target

\begin{tabular}{||c|c|c|c|c||}
\hline$k \rightarrow k+1$ & $\mathrm{VS}$ & $\mathrm{S}$ & $\mathrm{B}$ & $\mathrm{VB}$ \\
\hline $\mathrm{VS}$ & 1 & 1 & 0.15 & 0.02 \\
\hline $\mathrm{S}$ & 0.15 & 1 & 1 & 0.15 \\
\hline $\mathrm{B}$ & 0.02 & 0.15 & 1 & 1 \\
\hline $\mathrm{VB}$ & 0 & 0.02 & 0.15 & 1 \\
\hline
\end{tabular}

\begin{tabular}{||c|c|c|c|c||}
\hline$k \rightarrow k+1$ & VS & S & B & VB \\
\hline VS & 1 & 0.15 & 0.02 & 0.0 \\
\hline S & 1 & 1 & 0.15 & 0.02 \\
\hline B & 0.15 & 1 & 1 & 0.15 \\
\hline VB & 0.02 & 0.15 & 1 & 1 \\
\hline
\end{tabular}

These fuzzy relations represent the degree of possibility for associations between respective (input, output) pairs. Then, we are able to realize our models' based filters running in parallel.

\subsection{Models' Conditioned Amplitude State Tendency Prediction}

At initial moment $k$ the target is characterized by the fuzzified amplitude values according to the models $\mu_{A^{A} p p}(k / k)$ and $\mu_{A^{R} e c}(k / k)$. Using these fuzzified amplitudes and applying the described above Zadeh max-min compositional rule equation( 5) to relation 1 - $A p p(k \rightarrow k+1)$ - and relation $2-\operatorname{Rec}(k \rightarrow k+1)$, we obtain models conditioned amplitude state tendency for time moment $k+1$, i.e.:

$$
\begin{aligned}
& \mu_{A^{A} p p}(k+1 / k)=\max \left(\min \left(\mu_{A^{A} p p}(k / k), \mu_{A} p p(k \rightarrow k+1)\right)\right), \\
& \mu_{A^{R} e c}(k+1 / k)=\max \left(\min \left(\mu_{A^{R_{e c}}}(k / k), \mu_{R} e c(k \rightarrow k+1)\right)\right) .
\end{aligned}
$$

\subsection{Weighting Procedure for Noise Reduction}

In order to reduce the influence of measurement noise over the amplitude tendency prediction, a weighting procedure is applied to make the measurement more informative. This procedure can be considered as an adaptive linear combiner as follows:

- We compute the degree to which the new fuzzified measurement intersects each of the linguistic terms in the frame $\Theta=\{V S, S, B, V B\}$. Actually, in that way we consider the likelihoods of receiving particular observation on condition that it 
originates from each of these terms, i.e.:

$$
L_{i}(A(k+1) / \Theta(i))=h g t[A(k+1) \cap \Theta(i)]=\sup \left\{\min \left(\mu_{A(k+1)}, \mu_{\Theta(i)}\right)\right\}, i=1 \div 4,
$$

where the operator hgt denotes the height of a resulting fuzzy sets, obtained after intersection between fuzzified new amplitude value and membership function of each of the linguistic terms in the frame $\Theta$;

- Using these likelihoods as respective weighting coefficients, we form the convex combination of the linguistic terms. Thus we take into account the degree of their influence over the received measurement. A normalization procedure is applied. The new fuzzy set represents the weighted measurement with a following membership function:

$\mu_{A^{W}}(x)=\sum_{i} L_{i}^{N} \cdot \mu_{\Theta(i)}$, where $L_{i}^{N}=L_{i} / \Sigma L_{i} ; L_{i}^{N} \geq 0 ; \sum_{i} L_{i}^{N}=1$.

Example.

At scan 4 the new crisp amplitude measurement is $A=0.7487$.

- After applying fuzzification procedure one obtains:

$\mu_{V S}(A)=0.0 ; \quad \mu_{S}(A)=0.0189 ; \mu_{B}(A)=0.7854 ; \mu_{V B}(A)=0.0373$.

- Bearing in mind the a priori defined input feature frame $\theta$, it is possible to define: $L_{1}(A / V S)=h g t[A \cap V S]=\max \left\{\min \left(\mu_{A}, \mu_{V S}\right)\right\}=$

$=\max \{\min (0,1), \min (0.0189,0.15), \min (0.7854,0), \min (0.0373,0)\}=0.0189$.

- The application of the above procedure according to the other linguistic values yields: $L_{2}(A / S)=0.15 ; L_{3}(A / B)=0.7854, L_{4}(A / V B)=0.15$.

- A normalization procedure is applied to $L_{i}: L_{i}^{N}=L_{i} / \Sigma L_{i}, \quad i=1 \div 4$. $\quad$ It yields: $L_{1}^{N}=0.0172 ; \quad L_{2}^{N}=0.1358 ; \quad L_{3}^{N}=0.7112 ; \quad L_{4}^{N}=0.1358$.

- The weighted measurement is formed as a convex combination: $\mu_{A}^{W}=L_{1}^{N} *$ $\mu_{V S}+L_{2}^{N} * \mu_{S}+L_{3}^{N} * \mu_{B}+L_{4}^{N} * \mu_{V B}$. As a result, we obtain $\mu_{V S}\left(A^{W}\right)=0.0499$; $\mu_{S}\left(A^{W}\right)=0.3259 ; \quad \mu_{B}\left(A^{W}\right)=1.0 ; \mu_{V B}\left(A^{W}\right)=0.3225$.

\subsection{Updating State Estimates}

The updated states are obtained through a fuzzy set intersection between the weighted new measurement and corresponding modes conditioned amplitude state predictions:

$$
\begin{aligned}
& \mu_{A^{A} p p}(k+1 / k+1)=\min \left(\mu_{A^{W}}, \mu_{A^{A} p p}(k+1 / k)\right), \\
& \mu_{A^{R} e c}(k+1 / k+1)=\min \left(\mu_{A^{W}}, \mu_{A^{R} e c}(k+1 / k)\right) .
\end{aligned}
$$

\section{Simulation Study}

A simulation scenario is developed for a simple target trajectory (Figure 4) in plane coordinates $(X, Y)$ and for constant velocity movement. The target's starting point and velocities are: $\left(X_{0}=5 \mathrm{~km}, Y_{0}=10 \mathrm{~km}\right), \dot{X}=100 \mathrm{~m} / \mathrm{s}, \dot{Y}=100 \mathrm{~m} / \mathrm{s}$ and 


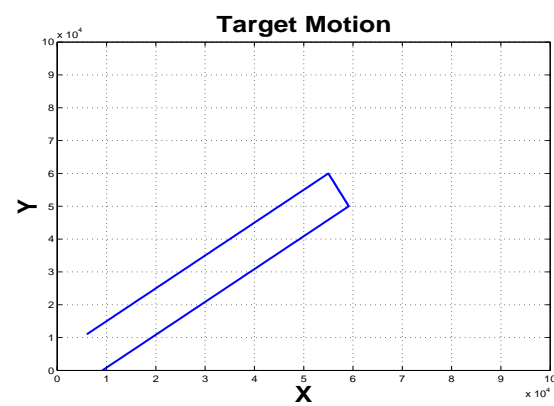

Figure 4: Target trajectory

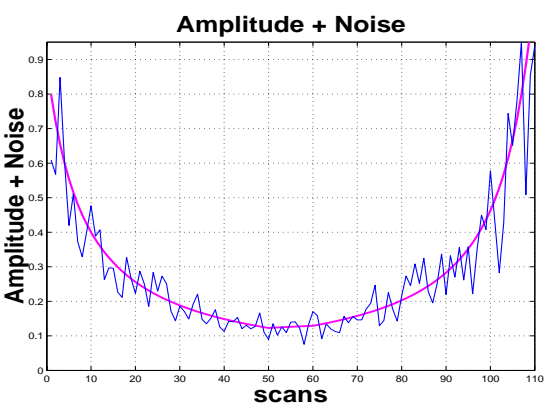

Figure 5: Measurements dynamics

$\dot{X}=-100 \mathrm{~m} / \mathrm{s}, \dot{Y}=-100 \mathrm{~m} / \mathrm{s}$. The time sampling rate is $T=5 \mathrm{~s}$. The dynamics of target movement is modeled by simple equations:

$$
x(k)=x(k-1)+\dot{x} \cdot T ; \quad y(k)=y(k-1)+\dot{y} \cdot T .
$$

The amplitude value $Z_{A}(k)=A(k)+\nu_{A}(k)$ measured by passive radar is a random Gaussian distributed process (Figure 5) with mean $A(k)=1 / D(k)$ and covariance $\sigma_{A}(k)=0.3 \cdot \operatorname{rand}(1,1) / D(k) . D(k)=\sqrt[2]{x(k)^{2}+y(k)^{2}}$ is the distance to the target, $\{x(k), y(k)\}$ is the corresponding vector of coordinates, and $\nu_{A}(k)$ is the measurement noise. Each amplitude value (true one and the corresponding noisy one) received at time (scan) $k=1,2, \ldots$ is processed according to the block diagram of our target's behavior tracking system (Figure 1 ).

Figures 6-10 show the results obtained during the whole motion of the observed target (descending and approaching directions). They represent the tendency in target behavior, which is described via the time (scan) consecutive transitions of amplitude value $V B \rightarrow V B \rightarrow B \rightarrow B \rightarrow S \rightarrow S \rightarrow V S \rightarrow V S$ and respectively $V S \rightarrow V S \rightarrow S \rightarrow S \rightarrow$ $B \rightarrow B \rightarrow V B \rightarrow V B$. Figure 6 represents the case, when the measured amplitude values are without measurement's noise, i.e. $Z_{A}(k)=A(k)$. Two models - Approaching and Receding are maintained in parallel.

With the implementation of the developed algorithm (Figure 1) it becomes possible to make a correct decision about the plausibility of the considered models. It could be seen that between scans 1 and 90 target motion estimation is supported by the correct, for that case, Descending model. In the same time, the Approaching model has no reaction to the measurements dynamics, because it does not match the real target behavior Receding. Taking into account Figure 5, the amplitude measurements dynamics between scans 10 and 90 could be analyzed as relatively weak from the point of view of the fuzzification interface (Figure 2). Such a transition area is contingent on the assumed possibility for sojourning time, when the measured amplitude values during consecutive scans consistently reside in one and the same regions of that interface. It 


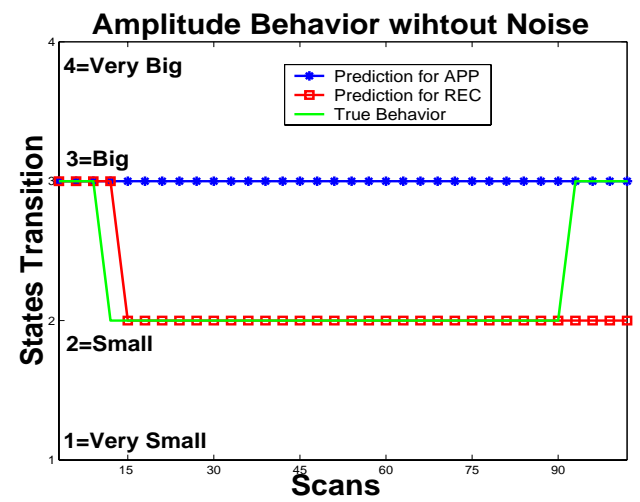

Figure 6: Target behavior estimation (without measurement noise)

Case 1: Amplitude Noise with $\sigma_{A}=0.2 \cdot \operatorname{rand}(1,1) / D(k)$

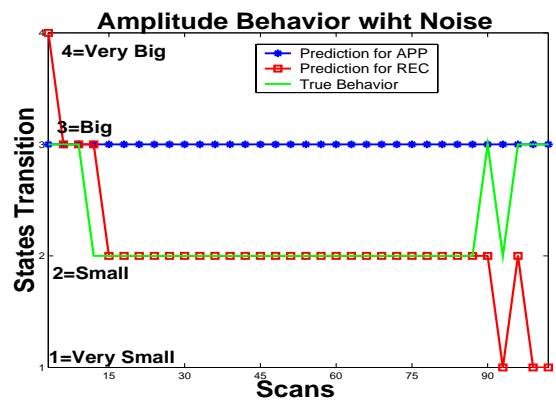

Figure 7: Target behavior estimation in case of noise.

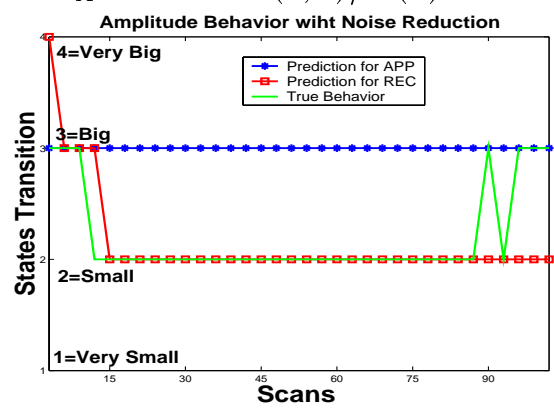

Figure 8: Target behavior estimation in case of noise reduction.

is characterized with a latency delay before switching to the opposite behavior mode. After scan 90 and until scan 115 it is obvious that the Descending model misses the amplitude changes, while the Approaching model becomes the plausible one. Figure 7 represents the case, when the measured amplitude values are corrupted by noise with $\sigma_{A}=0.2 \cdot \operatorname{rand}(1,1) / D(k)$.

Some disorder and discrepancy between predicted behavior tendency and true amplitude behavior take place, and it is difficult to make a firm decision about the tendency of target behavior. As presented on Figure 8, the application of the noise reduction procedure produces a 'smoothed' predicted behavior tendency, and it becomes possible to make a robust decision on the tendency of target behavior. The effect of that procedure is even more important when input measurements are corrupted by higher noise levels, 


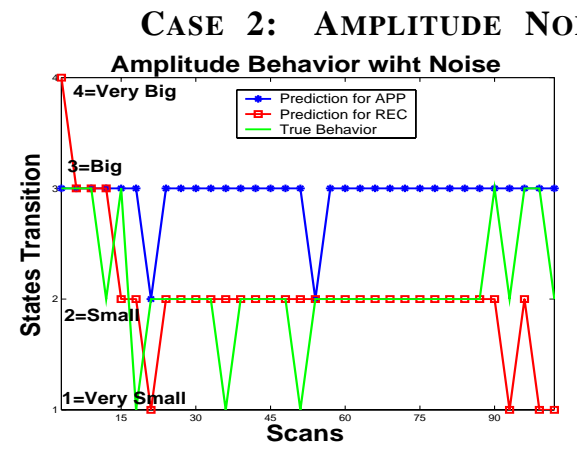

Figure 9: Target behavior estimation in case of noise.

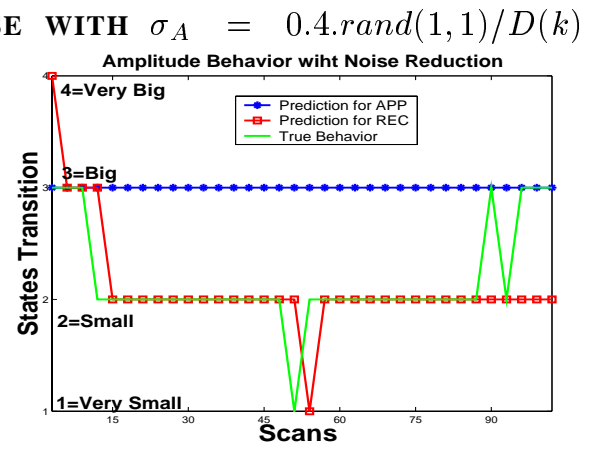

Figure 10: Target behavior estimation in case of noise reduction.

for example with $\sigma_{A}=0.4 \operatorname{rand}(1,1) / D(k)$ (Figure 9). In that case, some chaotic behavior is detected. In such critical situations the noise reduction procedure assures a more consistent process of amplitude tendency prediction (Figure 10).

\section{Conclusions}

An approach to estimating the tendency of target behavior was proposed and evaluated. It is based on Fuzzy Logic principles applied to conventional passive radar measurements. A particular real-time algorithm was developed. It was evaluated using computer simulation. Dealing simultaneously with numerical and linguistic data, an opportunity for robust reasoning is realized. The application of an additional weighting procedure for noise reduction improves the overall process of estimating the tendency of target behavior. The developed algorithm is suitable and adapted for processing noisy amplitude measurements. It entails modest computational load and provides simple and robust decisions about tendencies in target behavior. The proposed approach is suitable for obtaining a tactical picture for complex or ill-defined problems in engineering applications.

\section{Acknowledgement}

This research work was partially supported by the Center of Excellence BIS21 Grant ICA1-2000-70016.

\section{Notes}

1 Samuel S. Blackman and Robert Populi, Design and Analysis of Modern Tracking Systems (Norwood, MA: Artech House, 1999).

2 Yaakov Bar-Shalom, ed., Multitarget-Multisensor Tracking: Advanced Application (Nor- 
wood, MA: Artech House, 1990).

3 J.M. Mendel, "Fuzzy Logic Systems for Engineering: A Tutorial," Proc. of the IEEE 83, 3 (March 1995): 345-377.

4 Lotfi A. Zadeh, "From Computing with Numbers to Computing with Words - From Manipulation of Measurements to Manipulation of Perceptions," IEEE Transactions on Circuits and Systems 45, 1 (January 1999): 105-119.

5 Lotfi A. Zadeh, "Fuzzy Sets as a Basis for a Theory of Possibility," Fuzzy Sets and Systems 1 (1978): 3-28.

ALBENA PLAMENOVA TCHAMOVA is assistant research professor at the Central Laboratory for Parallel Processing, Bulgarian Academy of Sciences. She received M.S. and Ph.D. degrees from the Technical University of Sofia, Bulgaria, in 1978 and 1998 respectively. E-mail: tchamova@bas.bg.

TZVETAN ATANASOV SEMERDJIEV see p.90 